\title{
TIM Lecture Series The Laboratory for Analytic Sciences: Developing the Art and Science of Analysis
}

\section{J. David Harris}

\author{
"Our goal is to combine tradecraft and technology." \\ We are examining not only the methodologies and \\ the tools - the systems and the machinery - that we \\ can create in hardware and software, but also the \\ critical-thinking skills and structured analytic \\ techniques that we can bring to bear when thinking \\ about what the key questions are, what the data \\ means to us, and how to make sense of it.
}

J. David Harris

Director, Laboratory for Analytic Sciences (LAS)

\section{Overview}

The TIM Lecture Series is hosted by the Technology Innovation Management program (TIM; carleton.ca/tim) at Carleton University in Ottawa, Canada. The lectures provide a forum to promote the transfer of knowledge between university research to technology company executives and entrepreneurs as well as research and development personnel. Readers are encouraged to share related insights or provide feedback on the presentation or the TIM Lecture Series, including recommendations of future speakers.

The fifth TIM lecture of 2014 was held at Carleton University on July 8th and was presented by David Harris, Director of the Laboratory for Analytic Sciences (LAS; ncsu-las.org), a government, academic, and industry collaboration whose mission is to imagine, investigate, and implement innovative classified and unclassified solutions for a variety of tactical and strategic analytic challenges, including those related to cybersecurity.

\section{Summary}

The goal of the lecture was to share the early experiences and lessons learned in the Laboratory for Analytic Sciences and gather feedback from those who may have similar experiences or have faced analogous challenges. In the first part of the lecture, Harris provided a brief introduction to the laboratory and its analysis framework.
In the second part of the lecture, he discussed the lab's current work and demonstrated a collaboration tool they are using to both help improve the lab's efficiency and enhance its analytic approach.

\section{About the laboratory}

The activities of the laboratory are centred on the development of a science of analysis to address both nearterm problems and long-term strategic challenges of critical importance. Thus, the lab's work includes a strong research dimension, but it is also tasked with solving real-world problems that are affecting the community today. Cybersecurity is an area of focus, but the research and methodologies are applicable to a wide range of analogous domains, including healthcare, financial services, energy, agriculture, and retail, among many others.

Although the laboratory is based on a campus of North Carolina State University (Figure 1), it is the physical working space for a consortium of members from government, academia, and industry who bring a diversity of educational backgrounds, work roles, and experiences. Only about half of the people have backgrounds in science, technology, engineering, or math (STEM) in recognition of the broad dimensions of the challenges that need to be addressed, which extend well beyond the STEM fields and which will require non-technical innovation and input. Co-locating diverse staff from the various consortium members encourages interaction 


\section{TIM Lecture Series - The Laboratory for Analytic Sciences}

\section{J. David Harris}

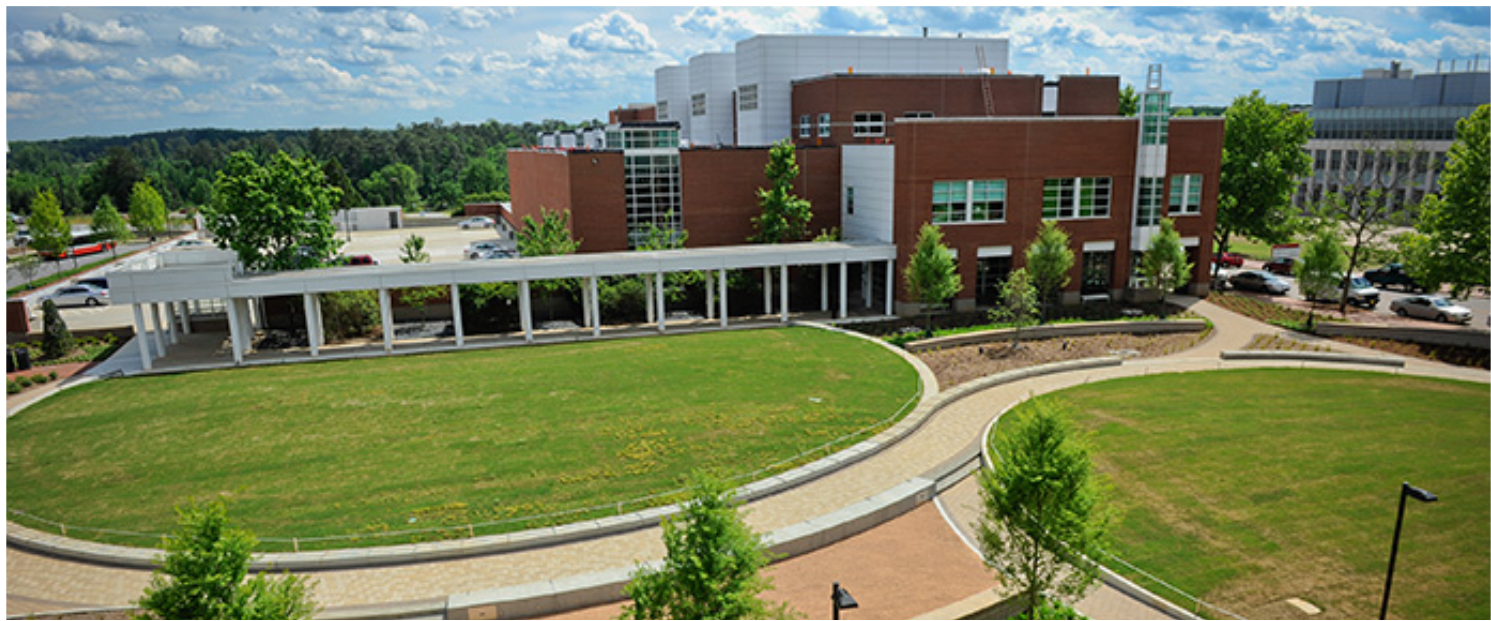

Figure 1. The Laboratory for Analytic Science on the campus of North Carolina State University

and collaboration between individuals who may bring their own unique and innovative approaches to problems. Working groups can be easily assembled and disassembled as work on particular challenges progresses.

Having completed its "ideation" phase, the laboratory is approximately one year through its initial three-year timeline and is currently in its "commit" phase. The laboratory currently houses 17 staff, with plans to expand to around 100 once it reaches its "validation" and "scale" phases.

\section{The LAS analysis framework}

The analysis framework under development at the LAS is based on the simultaneous need to look backwards with global awareness (i.e., making sense off a massive amount of "big data" from many sources) and to look forwards with strategic foresight (i.e., trying to anticipate where the currents within the stream of data will be going next). The framework depends on three ongoing activities:

1. Reflecting on the past: taking into account what we know about the data based on hindsight; saving and structuring past events

2. Observing the present: identifying events of interest, deciding which are more or less important, focusing efforts on specific areas within the sea of data

3. Imagining the future: making predictions, prioritizing where to look for future events that may form part of a potential narrative of events, developing "devil's advocate" predictions interpretations or assumptions are flawed
These activities are not only performed with past-to-future direction in mind; later events can inform our interpretations and activities about past events. For example, looking back at what actually happened can highlight missing pieces of the story about which we failed to collect data or about which we need to refine our interpretation. And ongoing back-and-forth between our interpretations of past, present, and future is the only way to fill in the picture and improve our capabilities for predicting events based on massive amounts of unstructured data. The overall goal is to improve both narrative processing (i.e., the telling of the story in a compelling and meaningful way) and analytic workflow (i.e., improving our ability to take meaningful actions in response to the data).

\section{Demonstration: Collaboration tools in the laboratory}

Finally, Harris demonstrated an online collaboration tool that is currently in use within the laboratory. Based in part on Yammer (yammer.com), a "private enterprise social network", the collaboration tool helps lab members interact, build relationships, and take advantage of the multidisciplinary approach. All of the entries about the activities of individuals are tagged so the data can be mined later, and the overall platform integrates various other tools (e.g., 4square, OSX, Google Glass), including automated event recording and interpretation, and other inputs. Thus, the tool not only contributes to the efficiency of the lab, but through its use, the lab is assembling a large and structured dataset with which it can test and refine its analytic framework and approach. The goal is to collect and mine data, then build stories (both imagined and observed), that can enable meaningful actions. 


\section{TIM Lecture Series - The Laboratory for Analytic Sciences}

\section{J. David Harris}

\section{About the Speaker}

J. David Harris is the inaugural Director of the Laboratory for Analytic Sciences in Raleigh, North Carolina, where the aim is to develop a science of analysis and analytic methodology. During nearly 25 years service with the U. S. Department of Defense, David has worked in a variety of technical and leadership positions in areas of research and development, technology transfer, and operations.
Citation: Harris, D. 2014. TIM Lecture Series - The Laboratory for Analytic Sciences: Developing the Art and Science of Analysis. Technology Innovation Management Review, 4(7): 52-54.

http://timreview.ca/article/813

Keywords: analysis, analytics, collaboration, big data, cybersecurity, instrumentation, monitoring, prediction, innovation, strategy, framework

This report was written by Chris McPhee. 